\title{
FUNCTIONS WITH BOUNDED SPECTRUM
}

\author{
HA HUY BANG
}

\begin{abstract}
Let $0<p \leq \infty, f(x) \in L_{p}\left(\mathbb{R}^{n}\right)$, and supp $F f$ be bounded, where $F$ is the Fourier transform. We will prove in this paper that the sequence $\left\|D^{\alpha} f\right\|_{p}^{1 /|\alpha|}, \alpha \geq 0$, has the same behavior as the sequence $\sup _{\xi \in \operatorname{supp} F f}\left|\xi^{\alpha}\right|^{1 /|\alpha|}$, $\alpha \geq 0$. In other words, if we know all "far points" of supp $F f$, we can wholly describe this behavior without any concrete calculation of $\left\|D^{\alpha} f\right\|_{p}, \alpha \geq 0$. A Paley-Wiener-Schwartz theorem for a nonconvex case, which is a consequence of the result, is given.
\end{abstract}

\section{INTRODUCTION}

The following result showing a relation between behavior of the sequence of norms of derivatives of a function and the support of its Fourier transform [2] has been proved: Let $1 \leq p \leq \infty$ and $D^{m} f(x) \in L_{p}\left(\mathbb{R}^{1}\right), m=0,1, \ldots$. Then there always exists the limit

$$
d_{f}=\lim _{m \rightarrow \infty}\left\|D^{m} f\right\|_{p}^{1 / m}
$$

and moreover

$$
d_{f}=\sup \{|\xi|: \xi \in \operatorname{supp} \tilde{f}\},
$$

where $\tilde{f}(\xi)=F f(\xi)$ is the Fourier transform of the function $f(x)$.

This result is of value in the theory of Sobolev spaces of infinite order, in particular, in studying imbedding theorems for Sobolev spaces of infinite order [3-5].

The question arises as to what happens for the $n$-dimensional case? In this paper we give a complete answer to this question. It should be noted that here we do not assume any restriction on geometrical properties of supp $\tilde{f}$ (which is called the spectrum of $f$ ).

We will use the following standard notation: $\alpha=\left(\alpha_{1}, \ldots, \alpha_{n}\right) \in \mathbb{Z}_{+}^{n} ; D=$ $\left(D_{1}, \ldots, D_{n}\right) ; D_{j}=\frac{\partial}{\partial x_{j}}, j=1, \ldots, n ; D^{\alpha}=D_{1}^{\alpha_{1}} \cdots D_{n}^{\alpha_{n}} ; \operatorname{sp}(f)=\operatorname{supp} \tilde{f}$.

Received by the editors December 16,1993; originally communicated to the Proceedings of the $A M S$ by Andrew Bruckner.

1991 Mathematics Subject Classification. Primary 26D10, 42B10, 46E30.

Key words and phrases. Inequalities for derivatives, Fourier transform.

Supported by the National Basic Research Program in Natural Science and by the NCSR of Vietnam "Applied Mathematics". 
And we presuppose that $0^{0}=\frac{0}{0}=1, \frac{\lambda}{0}=\infty$ for $\lambda>0, f(x) \in \mathscr{S}^{\prime}$, and $f(x) \not \equiv 0$.

\section{Results}

We will show the following

Theorem 1. Let $0<p \leq \infty, f(x) \in L_{p}\left(\mathbb{R}^{n}\right)$ and $\operatorname{sp}(f)$ be bounded. Then

$$
\lim _{|\alpha| \rightarrow \infty}\left(\left\|D^{\alpha} f\right\|_{p} / \sup _{\operatorname{sp}(f)}\left|\xi^{\alpha}\right|\right)^{1 /|\alpha|}=1 .
$$

To prove Theorem 1 we need the following results: Let $0<p \leq q \leq \infty$ and $K \subset \mathbb{R}^{n}$ be compact. Denote by $M_{K p}$ the class of all functions in $\mathscr{S}^{\prime} \cap L_{p}\left(\mathbb{R}^{n}\right)$ such that $\operatorname{sp}(f) \subset K$. The following Nikolsky inequality is well known the ([9], $[10$, p. 125]): There exists a constant $C(p, q, K)$ such that

$$
\|f\|_{q} \leq C(p, q, K)\|f\|_{p}
$$

for all $f \in M_{K p}$.

It follows from the Nikolsky inequality that $M_{K p} \subset M_{K \infty}, 0<p \leq \infty$.

Further, let $G$ be a domain in $\mathbb{R}^{n}$ and $m \in \mathbb{Z}_{+}$. Denote by $W_{m, 2}(G)$ the classical Sobolev space, i.e., the completion $C^{m}(G)$ with respect to the norm

$$
\|f\|_{m, 2}=\left(\sum_{|\alpha| \leq m}\left\|D^{\alpha} f\right\|_{L_{2}(G)}^{2}\right)^{1 / 2}
$$

And $W_{m, 2}^{0}(G)$ is the subspace of all functions $f(x) \in W_{m, 2}(G)$ such that the zero extension of $f(x)$ outside $G$ belongs to $W_{m, 2}\left(\mathbb{R}^{n}\right)$. For $s \in \mathbb{R}$, we put

$$
H_{(s)}=\left\{f \in \mathscr{S}^{\prime}:\|f\|_{(s)}=\left(\int_{\mathbb{R}^{n}}\left(1+|\xi|^{2}\right)^{s}|F f(\xi)|^{2} d \xi\right)^{1 / 2}<\infty\right\} .
$$

Then

$$
H_{(k)}=W_{k, 2}\left(\mathbb{R}^{n}\right) \quad \text { (topological imbedding) }
$$

if $k \in \mathbb{Z}_{+}$(see, for example, [1, p. 45; 6, p. 53; 7, 7.9.1]).

Proof of Theorem 1. We divide the proof into three cases.

Case $1(1 \leq p<\infty)$. We first establish the following inequality

$$
\varliminf_{|\alpha| \rightarrow \infty}\left(\left\|D^{\alpha} f\right\|_{p} /\left|\xi^{\alpha}\right|\right)^{1 /|\alpha|} \geq 1
$$

for any point $\xi \in \operatorname{sp}(f)$.

Actually, let $\xi^{0} \in \operatorname{sp}(f), \xi_{j}^{0} \neq 0, j=1, \ldots, n$. (It is easy to show later that there exist such points because of $p<\infty$.) For the sake of convenience, we assume that $\xi_{j}^{0}>0, j=1, \ldots, n$. We fix a number $0<\epsilon<\frac{1}{2} \min _{1 \leq j \leq n} \xi_{j}^{0}$ and choose a domain $G$ with a smooth boundary such that $\xi^{0} \in G$ and $G \subset\{\xi$ : $\left.\xi_{j}^{0}-\epsilon \leq \xi_{j} \leq \xi_{j}^{0}+\epsilon, j=1, \ldots, n\right\}$. Further we fix a function $\tilde{v}(\xi) \in C_{0}^{\infty}(G)$ such that $\xi^{0} \in \operatorname{supp}(\tilde{v} \tilde{f})$. Then

$$
\langle\tilde{v}(\xi) \tilde{f}(\xi), \tilde{w}(\xi)\rangle=\langle f(x), \varphi(x)\rangle,
$$


where $\tilde{w}(\xi) \in C_{0}^{\infty}(G)$ is an arbitrary function, $\varphi(x)=\stackrel{v}{v} * \stackrel{v}{w}(x)$, and $\stackrel{\vee}{u}(x)=$ $u(-x)$. The distribution $\tilde{v}(\xi) \tilde{f}(\xi)$ has a compact support; therefore, it can be represented in the form

$$
\tilde{v}(\xi) \tilde{f}(\xi)=\sum_{|\alpha| \leq m} D^{\alpha} h_{\alpha}(\xi),
$$

where $m$ is a nonnegative integer and $h_{\alpha}(\xi)$ are ordinary functions in $G$. Without loss of generality we may assume that $m \geq 2 n$. tion

It is well known that the Dirichlet problem for the elliptic differential equa-

$$
L_{2 m} \tilde{z}(\xi)=\sum_{|\alpha| \leq m}(-1)^{|\alpha|} D^{\alpha}\left(D^{\alpha} \tilde{z}(\xi)\right)=\tilde{v}(\xi) \tilde{f}(\xi)
$$

has a (unique) solution $\tilde{z}(\xi) \in W_{m, 2}^{0}(G)$ (see, for example, [6, p. 82]). Since (3), we obtain

$$
\left\langle\tilde{z}(\xi), L_{2 m} \tilde{w}(\xi)\right\rangle=\langle f(x), \varphi(x)\rangle
$$

for all $\tilde{w}(\xi) \in C_{0}^{\infty}(G)$. The left side of (4) admits a closure up to an arbitrary function $\tilde{w}(\xi) \in W^{0} m, 2(G)$. Hence, replacing $\tilde{w}(\xi)$ by $\xi^{\alpha} \tilde{w}(\xi)$, we get

$$
\left\langle\tilde{z}(\xi), L_{2 m}\left(\xi^{\alpha} \tilde{w}(\xi)\right)\right\rangle=(-i)^{|\alpha|}\left\langle D^{\alpha} f(x), \varphi(x)\right\rangle
$$

for all $\tilde{w}(\xi) \in W_{m, 2}^{0}(G)$.

Now let $\tilde{w}_{0}(\xi) \in W_{m, 2}^{0}(G)$ be the solution of the equation $L_{2 m} \tilde{w}_{0}(\xi)=\overline{\tilde{z}(\xi)}$. Since $0 \notin G$, we get

$$
L_{2 m}\left(\xi^{\alpha} \tilde{w}_{\alpha}(\xi)\right)=\prod_{j=1}^{n}\left(\xi_{j}^{0}-2 \epsilon\right)^{\alpha_{j}} \overline{\tilde{z}(\xi)},
$$

where $\tilde{w}_{\alpha}(\xi)=\prod_{j=1}^{n}\left(\xi_{j}^{0}-2 \epsilon\right)^{\alpha_{j}} \xi^{-\alpha} \tilde{w}_{0}(\xi)$ and $\alpha \geq 0$. Therefore, it follows from (5) that

$$
\prod_{j=1}^{n}\left(\xi_{j}^{0}-2 \epsilon\right)^{\alpha_{j}}\langle\tilde{z}(\xi), \overline{\tilde{z}(\xi)}\rangle \leq\left\|D^{\alpha} f\right\|_{p}\|v\|_{1}\left\|w_{\alpha}\right\|_{q},
$$

where $1 / p+1 / q=1$.

On the other hand, there exists a constant $C>0$ such that

$$
\|v\|_{1}\left\|w_{\alpha}\right\|_{q} \leq C, \quad \alpha \geq 0 .
$$

Indeed, let $|\beta| \leq 2 n$. Using

$$
x^{\beta} w_{\alpha}(x)=(-i)^{|\beta|} \prod_{j=1}^{n}\left(\xi_{j}^{0}-2 \epsilon\right)^{\alpha_{j}} \int_{G} e^{i x \xi} D^{\beta}\left(\xi^{-\alpha} \tilde{w}_{0}(\xi)\right) d \xi,
$$

the Leibniz formula, and the definition of $G$, we get

$$
\sup _{\mathbb{R}^{n}}\left|x^{\beta} w_{\alpha}(x)\right| \leq C_{1} \prod_{j=1}^{n}\left(\frac{\xi_{j}^{0}-2 \epsilon}{\xi_{j}^{0}-\epsilon}\right)^{\alpha_{j}} \sum_{\gamma \leq \beta}\left(\begin{array}{l}
\beta \\
\gamma
\end{array}\right) \prod_{k=1}^{n} \alpha_{k} \cdots\left(\alpha_{k}+\gamma_{k}-1\right),
$$

where

$$
C_{1}=\max \left\{\int_{G}\left|\xi^{-\gamma} D^{\beta-\gamma} \tilde{w}_{0}(\xi)\right| d \xi: \gamma \leq \beta,|\beta| \leq 2 n\right\}
$$


On the other hand, since

$$
\prod_{k=1}^{n} \alpha_{k} \cdots\left(\alpha_{k}+\gamma_{k}-1\right)<(|\alpha|+2 n)^{2 n}
$$

(because of $|\gamma| \leq|\beta| \leq 2 n$ ),

$$
2^{|\beta|}=\sum_{\gamma \leq \beta}\left(\begin{array}{l}
\beta \\
\gamma
\end{array}\right)
$$

and

$$
\lim _{|\alpha| \rightarrow \infty}(|\alpha|+2 n)^{2 n} \prod_{j=1}^{n}\left(\frac{\xi_{j}^{0}-2 \epsilon}{\xi_{j}^{0}-\epsilon}\right)^{\alpha_{j}}=0,
$$

we obtain

$$
\sup _{x \in \mathbb{R}^{n}}\left|x^{\beta} \omega_{\alpha}(x)\right| \leq C_{2}
$$

for all $|\beta| \leq 2 n$ and $\alpha \geq 0$. Therefore, there is an absolute constant $C_{3}$ such that

$$
\sup _{\mathbb{R}^{n}}\left(1+x_{1}^{2}\right) \cdots\left(1+x_{n}^{2}\right)\left|w_{\alpha}(x)\right| \leq C_{3}, \quad \alpha \geq 0 .
$$

So we have proved (7) with $C=C_{3} \pi^{n}\|v\|_{1}$. Combining (6) and (7) we obtain

$$
1 \leq \lim _{|\alpha| \rightarrow \infty}\left(\left\|D^{\alpha} f\right\|_{p} \prod_{j=1}^{n}\left(\xi_{j}^{0}-2 \epsilon\right)^{-\alpha_{j}}\right)^{1 /|\alpha|} .
$$

Therefore, since $\epsilon>0$ is arbitrarily chosen and

$$
\left[\prod_{j=1}^{n}\left(\frac{\xi_{j}^{0}-2 \epsilon}{\xi_{j}^{0}}\right)^{-\alpha_{j}}\right]^{1 /|\alpha|} \leq \max _{1 \leq j \leq n} \frac{\xi_{j}^{0}}{\xi_{j}^{0}-2 \epsilon},
$$

we obtain (2) (with $\xi=\xi^{0}$ ) by letting $\epsilon \rightarrow 0$.

Now we prove (2) for "zero points": Let $\xi^{0} \in \operatorname{sp}(f), \xi^{0} \neq 0$, and $\xi_{1}^{0} \cdots \xi_{n}^{0}=$ 0 . For the sake of convenience, we assume that $\xi_{j}^{0}>0, j=1, \ldots, k$, and $\xi_{k+1}^{0}=\cdots=\xi_{n}^{0}=0 \quad(1 \leq k<n)$. Then it is enough to show (2) only for indices $\alpha$ such that $\alpha_{k+1}=\cdots=\alpha_{n}=0$. Then the proof is analogous to the above one after the following modification of choosing $\epsilon$ : We fix a number $0<\epsilon<\frac{1}{2} \min _{1 \leq j \leq k} \xi_{j}^{0}$.

Second we prove that

$$
\varliminf_{|\alpha| \rightarrow \infty}\left(\left\|D^{\alpha} f\right\|_{p} / \sup _{\operatorname{sp}(f)}\left|\xi^{\alpha}\right|\right)^{1 /|\alpha|} \geq 1 .
$$

Assume the contrary, that there exists a subsequence $I_{1}$ such that

$$
\text { (I }) \lim _{|\alpha| \rightarrow \infty}\left(\left\|D^{\alpha} f\right\|_{p} / \sup _{\mathbf{s p}(f)}\left|\xi^{\alpha}\right|\right)^{1 /|\alpha|}<1,
$$


where the symbol $\left(I_{1}\right)$ in (9) means that we take the limit only for $\alpha \in I_{1}$. Then there exist a subsequence $I_{2} \subset I_{1}$ and numbers $0 \leq \beta_{j} \leq 1, j=1, \ldots, n$ such that $|\beta|=1$ and

$$
\text { (I2) } \lim _{|\alpha| \rightarrow \infty} \frac{\alpha_{j}}{|\alpha|}=\beta_{j}, \quad j=1, \ldots, n .
$$

Now we prove

$$
\lim _{\gamma \rightarrow \beta} \sup _{\operatorname{sp}(f)}\left|\xi^{\gamma}\right|=\sup _{\mathbf{s p}(f)}\left|\xi^{\beta}\right|
$$

if $\gamma \in \mathbb{R}_{+}^{n}$ and $\gamma \rightarrow \beta$.

Indeed, given $h>1$, there exists $\epsilon>0$ such that $h \gamma \geq \beta$ for $\gamma \in \mathbb{R}_{+}^{n}$ and $|\gamma-\beta| \leq \epsilon$. Further, let $|\xi| \leq M$ for all $\xi \in \operatorname{sp}(f)$. Then for $\xi \in \operatorname{sp}(f)$ and $\gamma \in \mathbb{R}_{+}^{n},|\gamma-\beta| \leq \epsilon$ we obtain

$$
\left|\xi^{\gamma}\right|=\left|\xi^{\gamma-\beta / h}\right|\left|\xi^{\beta}\right|^{1 / h} \leq M^{|\gamma-\beta / h|} \sup _{\operatorname{sp}(f)}\left|\xi^{\beta}\right|^{1 / h}
$$

Therefore

$$
\varlimsup_{\gamma \rightarrow \beta} \sup _{\mathbf{s p}(f)}\left|\xi^{\gamma}\right| \leq M^{|\beta|(1-1 / h)} \sup _{\operatorname{sp}(f)}\left|\xi^{\beta}\right|^{1 / h} .
$$

Letting $h \rightarrow 1$, we get

$$
\varlimsup_{\gamma \rightarrow \beta} \sup _{\operatorname{sp}(f)}\left|\xi^{\gamma}\right| \leq \sup _{\operatorname{sp}(f)}\left|\xi^{\beta}\right| .
$$

To prove (11) it remains to show that

$$
\varliminf_{\gamma \rightarrow \beta} \sup _{\operatorname{sp}(f)}\left|\xi^{\gamma}\right| \geq \sup _{\operatorname{sp}(f)}\left|\xi^{\beta}\right| .
$$

Let $\xi^{*} \in \operatorname{sp}(f)$ such that $\left|\xi^{* \beta}\right|=\sup _{\mathbf{s p}(f)}\left|\xi^{\beta}\right|$. Then it follows from $p<\infty$ that the distribution $\tilde{f}(\xi)$ cannot concentrate on the hyperplanes $\xi_{j}=0, j=1, \ldots, n$ (this fact will be shown later). Therefore, $\left|\xi^{* \beta}\right|>0$. Furthermore, let $\eta$ be an arbitrary point of $\operatorname{sp}(f)$. Then we will show later that the restriction of the distribution $\tilde{f}(\xi)$ on any neiborhood of $\eta$ also does not concentrate on the hyperplanes $\xi_{j}=0, j=1, \ldots, n$. Therefore, there exists a sequence $m \xi \in \mathrm{sp}(f), m \geq 1$, such that $m \xi_{j} \neq 0, j=1, \ldots, n$, for any $m \geq 1$ and $m \xi \rightarrow \xi^{*}, m \rightarrow \infty$. Then

for any $m \geq 1$. Hence,

$$
\sup _{\mathbf{s p}(f)}\left|\xi^{\gamma}\right| \geq\left.\right|_{m} \xi^{\gamma} \mid
$$

$$
\varliminf_{\gamma \rightarrow \beta} \sup _{\operatorname{sp}(f)}\left|\xi^{\gamma}\right| \geq\left.\varliminf_{\gamma \rightarrow \beta}\right|_{m} \xi^{\gamma}|=|_{m} \xi^{\beta} \mid .
$$

Letting $m \rightarrow \infty$, we obtain (12) and then (11).

Further, given $\lambda>1$, there is a number $k \geq 1$ such that $\left.\lambda\right|_{k} \xi^{\beta}|\geq| \xi^{* \beta} \mid$. Therefore, it follows from (10)-(11) and (2) that

(I $) \varliminf_{|\alpha| \rightarrow \infty}\left(\left\|D^{\alpha} f\right\|_{p} / \sup _{\operatorname{sp}(f)}\left|\xi^{\alpha}\right|\right)^{1 /|\alpha|}=\left(I_{2}\right) \varliminf_{|\alpha| \rightarrow \infty}\left\|D^{\alpha} f\right\|_{p}^{1 /|\alpha|} /\left|\xi^{* \beta}\right|$

$$
\begin{aligned}
& \geq\left(I_{2}\right) \frac{1}{\lambda} \varliminf_{|\alpha| \rightarrow \infty}\left\|D^{\alpha} f\right\|_{p}^{1 /|\alpha|} /\left|\xi_{k} \xi^{\beta}\right| \\
& =\left(I_{2}\right) \frac{1}{\lambda} \varliminf_{|\alpha| \rightarrow \infty}\left\|D^{\alpha} f\right\|_{p} /\left.\left.\right|_{k} \xi^{\alpha}\right|^{1 /|\alpha|} \geq \frac{1}{\lambda} .
\end{aligned}
$$


This contradicts (9) by letting $\lambda \rightarrow 1$. Thus we have proved (8).

Finally, we will show

$$
\varlimsup_{|\alpha| \rightarrow \infty}\left(\left\|D^{\alpha} f\right\|_{p} / \sup _{\operatorname{sp}(f)}\left|\xi^{\alpha}\right|\right)^{1 /|\alpha|} \leq 1 .
$$

We fix a domain $G \supset \operatorname{sp}(f)$ and a function $\psi \in C_{0}^{\infty}(G)$ such that $\psi(\xi)$ equals 1 in some neighborhood of $\operatorname{sp}(f)$. Further, let $0<q \leq 1$. We put $h_{\alpha}(\xi)=\psi(\xi) \xi^{\alpha}, \alpha \geq 0$. Then it follows from Hölder's inequality that for any $s>n(1 / q-1 / 2)$

$$
\begin{aligned}
\left\|F^{-1} h_{\alpha}\right\|_{q}^{q} & =\int\left(\left|\tilde{h}_{\alpha}(\xi)\right|^{2}\right)^{q / 2} d \xi \\
& \leq\left(\int\left|\tilde{h}_{\alpha}(\xi)\right|^{2}\left(1+|\xi|^{2}\right)^{s} d \xi\right)^{q / 2} \times\left(\int\left(1+|\xi|^{2}\right)^{-s q /(2-q)} d \xi\right)^{1-q / 2} .
\end{aligned}
$$

Therefore,

$$
\left\|F^{-1} h_{\alpha}\right\|_{q} \leq C^{\prime}\left\|h_{\alpha}\right\|_{(s)},
$$

where $C^{\prime}=C^{\prime}(s, q)$ is independent of $h_{\alpha}$.

Combining (14), the topological equality $H_{(k)}=W_{k, 2}\left(\mathbb{R}^{n}\right)$, and

$$
\left\|D^{\alpha} f\right\|_{p}=\left\|F^{-1}\left(\psi(\xi) \xi^{\alpha}\right) * f\right\|_{p} \leq\left\|F^{-1}\left(\psi(\xi) \xi^{\alpha}\right)\right\|_{1}\|f\|_{p},
$$

we get

$$
\left\|D^{\alpha} f\right\|_{p} \leq C\left\|\psi(\xi) \xi^{\alpha}\right\|_{k, 2}\|f\|_{p}, \quad \alpha \geq 0,
$$

where $C$ is independent of $f$ and $\alpha$ and $k=\left[\frac{n}{2}\right]+1$.

Given the Leibniz formula we get a constant $C_{1}=C_{1}(\psi, k)$ such that

$$
\left\|\psi(\xi) \xi^{\alpha}\right\|_{k, 2} \leq C_{1}|\alpha|^{k} \sup \left\{\sup _{G}\left|\xi^{\alpha-\gamma}\right|: \gamma \leq \alpha,|\gamma| \leq k\right\}, \quad \alpha \geq 0 .
$$

On the other hand, by an argument analogous to the previous one, we get

$$
\lim _{|\alpha| \rightarrow \infty}\left(\sup _{G}\left\{\sup _{G}\left|\xi^{\alpha-\gamma}\right|: \gamma \leq \alpha,|\gamma| \leq k\right\}\right)^{1 /|\alpha|} / \sup _{G}\left|\xi^{\alpha}\right|^{1 /|\alpha|}=1 .
$$

Actually, assume the contrary, that there exist a subsequence $I_{1}$ and a number $\delta>1$ such that

$$
\sup \left\{\sup _{G}\left|\xi^{\alpha-\gamma}\right|^{1 /|\alpha|}: \gamma \leq \alpha,|\gamma| \leq k\right\} \geq \delta \sup _{G}\left|\xi^{\alpha}\right|^{1 /|\alpha|}, \quad \alpha \in I_{1} .
$$

Therefore, there are a subsequence $I_{2} \subset I_{1}$, numbers $0 \leq \beta_{j} \leq 1, j=$ $1, \ldots, n$, and an index $\gamma^{0},\left|\gamma^{0}\right| \leq k$, such that $|\beta|=1$ and

$$
\text { (I2) } \lim _{|\alpha| \rightarrow \infty} \frac{\alpha_{j}-\gamma_{j}^{0}}{|\alpha|}=\beta_{j}, \quad j=1, \ldots, n,
$$

and

$$
\sup \left\{\sup _{G}\left|\xi^{\alpha-\gamma}\right|^{1 /|\alpha|}: \gamma \leq \alpha,|\gamma| \leq k\right\}=\sup _{G}\left|\xi^{\alpha-\gamma^{0}}\right|^{1 /|\alpha|}
$$

for all $\alpha \in I_{2}$. Therefore, by an argument analogous to that used for the proof of (11), we get

$$
\text { (I2) } \lim _{|\alpha| \rightarrow \infty} \sup _{G}\left|\xi^{\alpha-\gamma^{0}}\right|^{1 /|\alpha|}=\left(I_{2}\right) \lim _{|\alpha| \rightarrow \infty} \sup _{G}\left|\xi^{\alpha}\right|^{1 /|\alpha|}=\sup _{G}\left|\xi^{\beta}\right|>0,
$$

which contradicts (18). Thus we have proved (17). 
Combining (15)-(17) we obtain

$$
\varlimsup_{|\alpha| \rightarrow \infty}\left\|D^{\alpha} f\right\|_{p}^{1 /|\alpha|} / \sup _{G}\left|\xi^{\alpha}\right|^{1 /|\alpha|} \leq 1 .
$$

Now we assume the contrary that (13) does not hold. Then there exist a subsequence $J$ and numbers $\lambda>1,0 \leq \beta_{j} \leq 1, j=1, \ldots, n$, such that $|\beta|=1$ and

$$
\begin{aligned}
& (J) \lim _{|\alpha| \rightarrow \infty}|| D^{\alpha} f \|_{p}^{1 /|\alpha|} / \sup _{\operatorname{sp}(f)}\left|\xi^{\alpha}\right|^{1 /|\alpha|}=\lambda, \\
& (J) \lim _{|\alpha| \rightarrow \infty} \frac{\alpha_{j}}{|\alpha|}=\beta_{j}, \quad j=1, \ldots, n .
\end{aligned}
$$

Therefore, given the validity of $(11)$ when $\operatorname{sp}(f)$ is replaced by $G$ (which can be proved analogously because $G$ is open) and (19) we get

$$
\sup _{G}\left|\xi^{\beta}\right| / \sup _{\operatorname{sp}(f)}\left|\xi^{\beta}\right| \geq \lambda
$$

for any domain $G \supset \operatorname{sp}(f)$, which is impossible because of $\sup \left|\xi^{\beta}\right|>0$. The proof of Case 1 is complete.

Case $2(p=\infty)$. This is the most complicated case. It should be noted that many facts used in the proof of Case 1 are false in this case (for example, equality (11)). We first prove that if $\sup _{\operatorname{sp}(f)}\left|\xi^{\alpha}\right|=0$, then $D^{\alpha} f(x) \equiv 0$ (for the same $\alpha$ ). Indeed, without loss of generality we may assume that $\alpha_{j} \neq 0, j=$ $1, \ldots, k$, and $\alpha_{k+1}=\cdots=\alpha_{n}=0 \quad(1 \leq k \leq n)$. Then the distribution $\tilde{f}(\xi)$ concentrates on the hyperplanes $\xi_{j}=0, j \in\{1, \ldots, k\}=I$.

For each $j \in I$ we put

$$
G_{j}=\left\{\xi \in \mathbb{R}^{n}: \xi_{i} \neq 0, i \in I \backslash\{j\}\right\} .
$$

Then $G_{j}$ is open. And let $\tilde{f}_{1}(\xi)$ be the restriction of $\tilde{f}(\xi)$ on $\underset{j \in I}{\cup} G_{j}$. Then using a partition of unity (see, for example, [7, Theorem 1.4.5]), we get

$$
\tilde{f}_{1}(\xi)=\sum_{j=1}^{k} \varphi_{j}(\xi) \tilde{f}(\xi)
$$

where $\varphi_{j}(\xi) \in C_{0}^{\infty}\left(G_{j}\right), j \in I$.

The distribution $\varphi_{j}(\xi) \tilde{f}(\xi)$ concentrates on the hyperplanes $\xi_{j}=0$. Therefore, taking account of a remark on Theorem 2.3.5 mentioned in Example 5.1.2 in [7], we get

$$
F^{-1}\left(\varphi_{j} \tilde{f}\right)(x)=\sum_{\ell=0}^{N} g_{\ell}\left(x_{1}, \ldots, x_{j-1}, x_{j+1}, \ldots, x_{n}\right) \cdot\left(-i x_{j}\right)^{\ell},
$$

where $N$ is the order of the distribution $\tilde{f}(\xi)(N<\infty$ because supp $\tilde{f}$ is compact), $\tilde{g}_{\ell}\left(\xi_{1}, \ldots, \xi_{j-1}, \xi_{j+1}, \ldots, \xi_{n}\right), \ell=0,1, \ldots, N$ - distributions with compact support.

On the other hand, we have

$$
\left\|F^{-1}\left(\varphi_{j} \tilde{f}\right)\right\|_{\infty}=\left\|F^{-1} \varphi_{j} * f\right\|_{\infty} \leq\left\|F^{-1} \varphi_{j}\right\|_{1}\|f\|_{\infty}<\infty .
$$


Therefore, (20) is possible only if $\ell=0$. Consequently, the function $F^{-1}\left(\varphi_{j} \tilde{f}\right)(x)$ is independent of $x_{j}$. Hence, since $\alpha_{j} \neq 0$, we get

$$
D^{\alpha} F^{-1}\left(\varphi_{j} \tilde{f}\right)(x) \equiv 0, \quad j \in I,
$$

and then $D^{\alpha} f_{1}(x) \equiv 0$. Therefore, to prove $D^{\alpha} f(x) \equiv 0$, it is enough to show that $D^{\alpha}\left(f-f_{1}\right)(x) \equiv 0$.

Further, let $\xi \in \mathbb{R}^{n} \backslash \bigcup_{j \in I} G_{j}$. Then there exist at least two indices $i, j \in I$ such that $\xi_{i}=\xi_{j}=0$. Therefore, the distribution $\tilde{f}(\xi)-\tilde{f}_{1}(\xi)$ will concentrate on the hyperplanes $\xi_{i}=\xi_{j}=0, i, j \in I, i \neq j$.

Let $i, j \in I, i \neq j$. We put

$$
G_{i j}=\left\{\xi \in \mathbb{R}^{n}: \xi_{\ell} \neq 0, \ell \in I \backslash\{i, j\}\right\} .
$$

Then $G_{i j}$ is open. And let $\tilde{f}_{2}(\xi)$ be the restriction of $\tilde{f}(\xi)-\tilde{f}_{1}(\xi)$ on the union of the sets $G_{i j}, i, j \in I, i \neq j$. Then using the partition of unity, we get

$$
\tilde{f}_{2}(\xi)=\sum_{i, j \in I, i \neq j} \varphi_{i j}(\xi)\left(\tilde{f}(\xi)-\tilde{f}_{1}(\xi)\right),
$$

where $\varphi_{i j}(\xi) \in C_{0}^{\infty}\left(G_{i j}\right)$.

The distribution $\varphi_{i j}(\xi)\left(\tilde{f}(\xi)-\tilde{f}_{1}(\xi)\right)$ concentrates on the hyperplane $\xi_{i}=$ $\xi_{j}=0$. Therefore, $D^{\alpha} F^{-1}\left[\varphi_{i j}\left(\tilde{f}-\tilde{f}_{1}\right)\right](x) \equiv 0$ because, as shown above, $F^{-1}\left(\varphi_{i j}\left(\tilde{f}-\tilde{f}_{1}\right)\right)(x)$ is independent of variables $x_{i}, x_{j}$. Consequently, $D^{\alpha} f_{2}(x) \equiv 0$. Therefore, to prove $D^{\alpha} f(x) \equiv 0$, it is enough to show that $D^{\alpha}\left(f-f_{1}-f_{2}\right)(x) \equiv 0$.

Now let $\xi \in \mathbb{R}^{n} \backslash \bigcup\left\{G_{i j}: i, j \in I, i \neq j\right\}$. Then there are at least three indices $i_{1}, i_{2}, i_{3} \in I$ such that $\xi_{i_{1}}=\xi_{i_{2}}=\xi_{i_{3}}=0$. Therefore, the distribution $\tilde{f}(\xi)-$ $\tilde{f}_{1}(\xi)-\tilde{f}_{2}(\xi)$ concentrates on the hyperplanes $\xi_{i_{1}}=\xi_{i_{2}}=\xi_{i_{3}}=0, i_{1}, i_{2}, i_{3} \in$ $I, i_{1} \neq i_{2} \neq i_{3}$.

Again for $i_{1}, i_{2}, i_{3} \in I, i_{1} \neq i_{2} \neq i_{3}$, we put

$$
G_{i_{1} i_{2} i_{3}}=\left\{\xi \in \mathbb{R}^{n}: \xi_{j} \neq 0, j \in I \backslash\left\{i_{1}, i_{2}, i_{3}\right\}\right\}
$$

and call $\tilde{f}_{3}(\xi)$ the restriction of $\tilde{f}(\xi)-\tilde{f}_{1}(\xi)-\tilde{f}_{2}(\xi)$ on the union of the sets $G_{i_{1} i_{2} i_{3}}, i_{1}, i_{2}, i_{3} \in I, i_{1} \neq i_{2} \neq i_{3}$. Then we have again $D^{\alpha} f_{3}(x) \equiv 0$. So it is enough to prove $D^{\alpha}\left(f-f_{1}-f_{2}-f_{3}\right)(x) \equiv 0$, where $\tilde{f}(\xi)-\tilde{f}_{1}(\xi)-\tilde{f}_{2}(\xi)-\tilde{f}_{3}(\xi)$ concentrates on the hyperplanes $\xi_{i_{1}}=\xi_{i_{2}}=\xi_{i_{3}}=\xi_{i_{4}}=0, i_{1}, i_{2}, i_{3}, i_{4} \in I, i_{1} \neq$ $i_{2} \neq i_{3} \neq i_{4}$. Repeating the above arguments, we obtain the distribution $\tilde{f}(\xi)-$ $\tilde{f}_{1}(\xi)-\cdots-\tilde{f}_{k-1}(\xi)$ which concentrates on the hyperlane $\xi_{1}=\cdots=\xi_{k}=0$ and $D^{\alpha} f(x) \equiv 0$ if $D^{\alpha}\left(f-f_{1}-\cdots-f_{k-1}\right)(x) \equiv 0$. The last fact is clear because, as shown above, $\left(f-f_{1}-\cdots-f_{k-1}\right)(x)$ does not depend on variables $x_{1}, \ldots, x_{k}$. Thus we have proved $D^{\alpha} f(x) \equiv 0$.

By the result just obtained, it is enough to show (1) only for multi-indices $\alpha \geq 0$ such that $\sup \left|\xi^{\alpha}\right|>0$ and denote by $P$ the set of all such as multiindices. $\operatorname{sp}(f)$

Second we notice that inequalities (2) and (19) have been proved for $1 \leq$ $p \leq \infty$.

Next we prove that

$$
\text { (P) } \varliminf_{|\alpha| \rightarrow \infty}\left(\left\|D^{\alpha} f\right\|_{\infty} / \sup _{\operatorname{sp}(f)}\left|\xi^{\alpha}\right|\right)^{1 /|\alpha|} \geq 1 .
$$


Assume to the contrary, that there exist a subsequence $I \subset P$, a number $\lambda<1$, and a vector $\beta \geq 0,|\beta|=1$ such that

$$
\begin{aligned}
& \text { (I) } \lim _{|\alpha| \rightarrow \infty}\left(|| D^{\alpha} f \|_{\infty} / \sup _{\mathbf{s p}(f)}\left|\xi^{\alpha}\right|\right)^{1 /|\alpha|}<\lambda, \\
& \text { (I) } \lim _{|\alpha| \rightarrow \infty} \frac{\alpha}{|\alpha|}=\beta .
\end{aligned}
$$

Note that

$$
\text { (I) } \varliminf_{|\alpha| \rightarrow \infty} \sup _{\operatorname{sp}(f)}\left|\xi^{\alpha}\right|^{1 /|\alpha|}>0 .
$$

Indeed, assume to the contrary, that there exists a subsequence $J \subset I$ such that

$$
(J) \lim _{|\alpha| \rightarrow \infty} \sup _{\operatorname{sp}(f)}\left|\xi^{\alpha}\right|^{1 /|\alpha|}=0 .
$$

For any $1 \leq k \leq n$ and $i_{1}, \ldots, i_{k} \in\{1, \ldots, n\}$ we put

$T_{i_{1} \ldots i_{k}}=\left\{\alpha \geq 0: \alpha_{i_{1}} \neq 0, \ldots, \alpha_{i_{k}} \neq 0\right.$ and $\alpha_{j}=0$ if $\left.j \notin\left\{i_{1}, \ldots, i_{k}\right\}\right\}$.

Then there exist $1 \leq k \leq n$ and $i_{1}, \ldots, i_{k} \in\{1, \ldots, n\}$ such that $J_{i_{1} \ldots i_{k}}=$ $J \cap T_{i_{1} \ldots i_{k}}$ is unbounded. Therefore, clearly, we get

$$
\left(J_{i_{1} \ldots i_{k}}\right) \underset{|\alpha| \rightarrow \infty}{\varliminf_{\operatorname{sp}(f)}} \sup \left|\xi^{\alpha}\right|^{1 /|\alpha|} \geq\left(J_{i_{1} \ldots i_{k}}\right) \varliminf_{|\alpha| \rightarrow \infty}\left|\eta^{\alpha}\right|^{1 /|\alpha|}>0,
$$

where $\eta$ is any point of $\operatorname{sp}(f)$ such that $\eta_{i_{1}} \neq 0, \ldots, \eta_{i_{k}} \neq 0$. This contradicts (25). So we have proved (24).

Further, let ${ }_{\alpha} \xi \in \operatorname{sp}(f):\left|{ }_{\alpha} \xi^{\alpha}\right|=\sup _{\operatorname{sp}(f)}\left|\xi^{\alpha}\right|$. Then ${ }_{\alpha} \xi_{i_{1}} \neq 0, \ldots,{ }_{\alpha} \xi_{i_{k}} \neq 0$ for any $\alpha \in J_{i_{1} \ldots i_{k}}$ and, by taking a subsequence, without loss of generality we may assume that for some $\xi^{*} \in \mathbf{s p}(f)$

$$
\left(J_{i_{1} \ldots i_{k}}\right) \lim _{|\alpha| \rightarrow \infty} \alpha \xi=\xi^{*} .
$$

Now we consider two cases of $\xi^{*}$ :

If $\xi_{i_{j}}^{*} \neq 0, j=1, \ldots, k$, then, obviously,

$$
\left(J_{i_{1} \ldots i_{k}}\right) \lim _{|\alpha| \rightarrow \infty}\left|\alpha \xi^{\alpha}\right|^{1 /|\alpha|}=\left|\xi^{* \beta}\right|=\left(J_{i_{1} \ldots i_{k}}\right) \lim _{|\alpha| \rightarrow \infty}\left|\xi^{* \alpha}\right|^{1 /|\alpha|}
$$

which together with $\xi^{*} \in \mathbf{s p}(f),(2)$, and (22) implies

$$
\begin{aligned}
1 & \leq\left(J_{i_{1} \ldots i_{k}}\right) \lim _{|\alpha| \rightarrow \infty}\left(\left\|D^{\alpha} f\right\|_{\infty} /\left|\xi^{* \alpha}\right|\right)^{1 /|\alpha|} \\
& =\left(J_{i_{1} \ldots i_{k}}\right) \lim _{|\alpha| \rightarrow \infty}\left(\left\|D^{\alpha} f\right\|_{\infty} / \sup _{\mathbf{s p}(f)}\left|\xi^{\alpha}\right|\right)^{1 /|\alpha|}<\lambda<1,
\end{aligned}
$$

which is impossible.

Otherwise, without loss of generality we may assume that $\xi_{i_{1}}^{*}=\cdots=\xi_{i_{m}}^{*}=0$ and $\xi_{i_{m+1}}^{*} \neq 0, \ldots, \xi_{i_{k}}^{*} \neq 0$ for some $1 \leq m \leq k$.

Since (24) and (26), it follows that $\xi^{*} \neq 0$, Therefore, $m<k$. Further, by virtue of $(23)-(24),(26)$, the definition of ${ }_{\alpha} \xi$, and $\xi_{i_{1}}^{*}=\cdots=\xi_{i_{m}}^{*}=0$ we obtain $\beta_{i_{1}}=\cdots=\beta_{i_{m}}=0$. Since, clearly,

$$
\begin{gathered}
\left(J_{i_{1} \ldots i_{k}}\right) \lim _{|\alpha| \rightarrow \infty}\left|\alpha \xi_{i_{m+1}}^{\alpha_{i_{m+1}}} \ldots \alpha \xi_{i_{k}}^{\alpha_{i_{k}}}\right|^{1 /|\alpha|}=\left|\xi_{i_{m+1}}^{{ }^{\beta_{i_{m+1}}}} \ldots \xi_{i_{k}}^{\beta_{i_{k}}}\right| \\
=\left(J_{i_{1} \ldots i_{k}}\right) \lim _{|\alpha| \rightarrow \infty} \mid \xi_{i_{m+1}{ }^{a_{m+1}}} \ldots \xi_{i_{k}}^{*^{a_{i_{k}}}} !^{1 /|\alpha|}
\end{gathered}
$$


there exist $\nu \in J_{i_{1} \ldots i_{k}}$ and $N>0$ such that

$$
\left|{ }_{\alpha} \xi_{i_{\ell}}\right| \leq \lambda^{-1}\left|{ }_{\nu} \xi_{i_{\ell}}\right|, \quad \ell=m+1, \ldots, k,
$$

for all $|\alpha| \geq N, \alpha \in J_{i_{1} \ldots i_{k}}$

On the other hand, it follows from ${ }_{\nu} \xi_{i_{1}} \neq 0, \ldots,{ }_{\nu} \xi_{i_{k}} \neq 0$ and

$$
\left(J_{i_{1} \ldots i_{k}}\right) \lim _{|\alpha| \rightarrow \infty} \xi_{i_{j}}=\xi_{i_{j}}^{*}=0, \quad j=1, \ldots, m,
$$

that there exists $M>0$ such that

$$
\left|{ }_{\alpha} \xi_{i_{j}}\right| \leq\left.\right|_{\nu} \xi_{i_{j}} \mid, \quad j=1, \ldots, m,
$$

for all $|\alpha| \geq M, \alpha \in J_{i_{1} \ldots i_{k}}$. This together with (27) implies

$$
\left|{ }_{\alpha} \xi_{i_{j}}\right| \leq \lambda^{-1}\left|{ }_{\nu} \xi_{i_{j}}\right|, \quad j=1, \ldots, k,
$$

for all $|\alpha| \geq \max \{M, N\}, \alpha \in J_{i_{1} \ldots i_{k}}$. Therefore,

$$
\sup _{\operatorname{sp}(f)}\left|\xi^{\alpha}\right|^{1 /|\alpha|}=\left|\alpha \xi^{\alpha}\right|^{1 /|\alpha|} \leq\left.\left.\lambda^{-1}\right|_{\nu} \xi^{\alpha}\right|^{1 /|\alpha|}
$$

which together with (2) and (22) implies

$$
\begin{aligned}
1 & \leq\left(J_{i_{1} \ldots i_{k}}\right) \varliminf_{|\alpha| \rightarrow \infty}\left(\left\|D^{\alpha} f\right\|_{\infty} /\left.\right|_{\nu} \xi^{\alpha} \mid\right)^{1 /|\alpha|} \\
& \leq\left(J_{i_{1} \ldots i_{k}}\right) \lambda^{-1} \lim _{|\alpha| \rightarrow \infty}\left(\left\|D^{\alpha} f\right\|_{\infty} / \sup _{\mathbf{s p}(f)}\left|\xi^{\alpha}\right|\right)^{1 /|\alpha|}<1 .
\end{aligned}
$$

We thus arrive at a contradiction. So we have proved (21).

Finally, to complete the proof it remains to show that

$$
(P) \varlimsup_{|\alpha| \rightarrow \infty}\left(\left\|D^{\alpha} f\right\|_{\infty} / \sup _{\operatorname{sp}(f)}\left|\xi^{\alpha}\right|\right)^{1 /|\alpha|} \leq 1 .
$$

Assume to the contrary, that there exist a subsequence $I \subset P$, a number $h>1$, and a vector $\beta \geq 0,|\beta|=1$ such that

$$
\begin{aligned}
& \text { (I) } \lim _{|\alpha| \rightarrow \infty}\left(\left\|D^{\alpha} f\right\|_{\infty} / \sup _{\operatorname{sp}(f)}\left|\xi^{\alpha}\right|\right)^{1 /|\alpha|}>h, \\
& \text { (I) } \lim _{|\alpha| \rightarrow \infty} \frac{\alpha}{|\alpha|}=\beta .
\end{aligned}
$$

Notation being as above, we have $1 \leq k \leq n$ and $i_{1}, \ldots, i_{k} \in\{1, \ldots, n\}$ such that $I_{i_{1} \ldots i_{k}}=I \cap T_{i_{1} \ldots i_{k}}$ is unbounded.

We put

$$
\begin{aligned}
Q=\left\{\eta \in \mathbb{R}^{n}:\right. & \exists\{m \xi\} \subset \operatorname{sp}(f),{ }_{m} \xi_{j} \neq 0, \\
& \left.j \in\left\{i_{1}, \ldots, i_{k}\right\}, m \geq 1, \lim _{m \rightarrow \infty} \xi=\eta\right\}, \\
& Q_{\delta}=\{x+y: x \in Q,|y|<\delta\}, \quad \delta>0,
\end{aligned}
$$

and $H=\mathbb{R}^{n} \backslash Q$. Then $Q$ is close, $H$ and $Q_{\delta}$ are open.

Therefore, since $\operatorname{sp}(f) \subset Q_{\delta} \cup H\left(=\mathbb{R}^{n}\right)$, we obtain

$$
\tilde{f}(\xi)=\varphi_{\delta}(\xi) \tilde{f}(\xi)+\psi(\xi) \tilde{f}(\xi), \quad \varphi_{\delta} \in C_{0}^{\infty}\left(Q_{\delta}\right), \psi \in C_{0}^{\infty}(H) .
$$

By an argument analogous to the previous one, we can prove that $D^{\alpha} F^{-1}(\psi \tilde{f})(x)$ $\equiv 0$ for all $\alpha \in I_{i_{1} \ldots i_{k}}$. Hence, it follows from (29) that

$$
\left(I_{i_{1} \ldots i_{k}}\right) \lim _{|\alpha| \rightarrow \infty}\left(\left\|D^{\alpha} F^{-1}\left(\varphi_{\delta} \tilde{f}\right)\right\|_{\infty} / \sup _{\operatorname{sp}(f)}\left|\xi^{\alpha}\right|\right)^{1 /|\alpha|}>h
$$

for any $\delta>0$. 
On the other hand, by an argument used in the proof of equality (11), we get

$$
\left(I_{i_{1} \ldots i_{k}}\right) \lim _{|\alpha| \rightarrow \infty} \sup _{Q_{\delta}}\left|\xi^{\alpha}\right|^{1 /|\alpha|}=\sup _{Q_{\delta}}\left|\xi^{\beta}\right| .
$$

Further, let ${ }_{m} \theta \in Q_{1 / m}:\left|{ }_{m} \theta^{\beta}\right|=\sup _{Q_{1 / m}}\left|\xi^{\beta}\right|, m \geq 1$. Then there exist a subsequence $\left\{m_{k}\right\}$ (for simplicity of notation we assume that $m_{k}=k, k \geq 1$ ) and a point $\theta^{*} \in Q$ such that $m \theta \rightarrow \theta^{*}, m \rightarrow \infty$. Then

$$
0<\sup _{Q}\left|\xi^{\beta}\right| \leq \lim _{m \rightarrow \infty}\left|\dot{m} \theta^{\beta}\right|=\left|\theta^{* \beta}\right| \text {. }
$$

Arguing as in the proof of (12) and taking account of $\theta^{*} \in Q$ and (30) we obtain

$$
\left|\theta^{* \beta}\right| \leq\left(I_{i_{1} \ldots i_{k}}\right) \varliminf_{|\alpha| \rightarrow \infty} \sup _{Q}\left|\xi^{\alpha}\right|^{1 /|\alpha|}
$$

Further, because inequality (19) was proved for $1 \leq p \leq \infty$, we have

$$
\left(I_{i_{1} \ldots i_{k}}\right) \varlimsup_{|\alpha| \rightarrow \infty}\left(\left\|D^{\alpha} F^{-1}\left(\varphi_{1 / m} \tilde{f}\right)\right\|_{\infty} / \sup _{Q_{1 / m}}\left|\xi^{\alpha}\right|\right)^{1 /|\alpha|} \leq 1
$$

for any $m \geq 1$.

Now we fix a number $m \geq 1$ such that $\left|{ }_{m} \theta^{\beta}\right| \leq h\left|\theta^{* \beta}\right|$. Then combining (31)-(34), we obtain

$$
\begin{aligned}
1 & \geq\left(I_{i_{1} \ldots i_{k}}\right) \varlimsup_{|\alpha| \rightarrow \infty}\left(\left\|D^{\alpha} F^{-1}\left(\varphi_{1 / m} \tilde{f}\right)\right\|_{\infty} / \sup _{Q_{1 / m}}\left|\xi^{\alpha}\right|\right)^{1 /|\alpha|} \\
& =\left(I_{i_{1} \ldots i_{k}}\right) \varlimsup_{|\alpha| \rightarrow \infty}\left\|D^{\alpha} F^{-1}\left(\varphi_{1 / m} \tilde{f}\right)\right\|_{\infty}^{1 /|\alpha|} /\left.\right|_{m} \theta^{\beta} \mid \\
& \geq\left(I_{i_{1} \ldots i_{k}}\right) \varlimsup_{|\alpha| \rightarrow \infty} h^{-1}|| D^{\alpha} F^{-1}\left(\varphi_{1 / m} \tilde{f}\right) \|_{\infty}^{1 /|\alpha|} /\left|\theta^{* \beta}\right| \\
& \geq\left(I_{i_{1} \ldots i_{k}}\right) \varlimsup_{|\alpha| \rightarrow \infty} h^{-1}\left(|| D^{\alpha} F^{-1}\left(\varphi_{1 / m} \tilde{f}\right) \|_{\infty} / \sup _{Q}\left|\xi^{\alpha}\right|\right)^{1 /|\alpha|} \\
& =\left(I_{i_{1} \ldots i_{k}}\right) \lim _{|\alpha| \rightarrow \infty} h^{-1}\left(\left\|D^{\alpha} F^{-1}\left(\varphi_{1 / m} \tilde{f}\right)\right\|_{\infty} / \sup _{\operatorname{sp}(f)}\left|\xi^{\alpha}\right|\right)^{1 /|\alpha|}>1,
\end{aligned}
$$

which is impossible.

The proof of Case 2 is complete.

Let us now return to prove the fact mentioned in the proof of Case 1 that the distribution $\tilde{f}(\xi)$ does not concentrate on the hyperplanes $\xi_{j}=0, j=1, \ldots, n$ if $1 \leq p<\infty$. Actually, assume to the contrary, that $\tilde{f}$ concentrates on the hyperplanes $\xi_{j}=0, j=1, \ldots, n$. Then, notation being as above, we have

$$
\tilde{f}_{1}(\xi)=\sum_{j=1}^{n} \varphi_{j}(\xi) \tilde{f}(\xi), \quad \varphi_{j} \in C_{0}^{\infty}\left(G_{j}\right)
$$

where $\tilde{f}_{1}$ is the restriction of $\tilde{f}$ on $\bigcup_{j=1}^{n} G_{j}$.

On the other hand, it follows from the Nikolsky inequality that $f(x) \in L_{\infty}$. Therefore, as shown above, $F^{-1}\left(\varphi_{j} \tilde{f}\right)(x)$ is independent of $x_{j}$, which is possible only if $F^{-1}\left(\varphi_{j} \tilde{f}\right)(x) \equiv 0$ because of

$$
\left\|F^{-1}\left(\varphi_{j} \tilde{f}\right)\right\|_{p} \leq\left\|F^{-1} \varphi_{j}\right\|_{1}\|f\|_{p}<\infty
$$


and $p<\infty$. Therefore, $\tilde{f}$ must concentrate on the hyperplanes $\xi_{i}=\xi_{j}=$ $0, i, j \in\{1, \ldots, n\}, i \neq j$. Repeating the above arguments, taking account of $p<\infty$, we obtain supp $\tilde{f} \subset\{0\}$, which is impossible because of $p<\infty$ and $f(x) \not \equiv 0$.

Further, let $\eta$ be an arbitrary point of $\operatorname{sp}(f)$. Then analogously we can prove that the restriction of $\tilde{f}(\xi)$ on any neighborhood of $\eta$ also cannot concentrate on the hyperplanes $\xi_{j}=0, j=1, \ldots, n$.

Case $3(0<p<1)$. The inequality

$$
\varliminf_{|\alpha| \rightarrow \infty}\left(\left\|D^{\alpha} f\right\|_{p} / \sup _{\operatorname{sp}(f)}\left|\xi^{\alpha}\right|\right)^{1 /|\alpha|} \geq 1
$$

follows from the Nikolsky inequality and Case 1 . The inverse inequality

$$
\varlimsup_{|\alpha| \rightarrow \infty}\left(\left\|D^{\alpha} f\right\|_{p} / \sup _{\operatorname{sp}(f)}\left|\xi^{\alpha}\right|\right)^{1 /|\alpha|} \leq 1
$$

can be proved in the same way as shown above with the following modification of $(15)$ :

$$
\left\|D^{\alpha} f\right\|_{p} \leq C\left\|\psi(\xi) \xi^{\alpha}\right\|_{k, 2}\|f\|_{p}, \quad \alpha \geq 0
$$

where $k=\left[n\left(\frac{1}{p}-\frac{1}{2}\right)\right]+1$.

Let us now prove (35). Given (14), we get

$$
\left\|F^{-1} h_{\alpha}\right\|_{p} \leq C^{\prime}\left\|h_{\alpha}\right\|_{(k)}, \quad \alpha \geq 0,
$$

where $C^{\prime}$ is independent of $f$ and $\alpha$ and notation is as above.

On the other hand, given

$$
\begin{aligned}
\operatorname{supp} & F\left(F^{-1} h_{\alpha}(.) f(x-.)\right) \subset \operatorname{supp} h_{\alpha}+\operatorname{supp} F(f(x-.)) \\
= & \operatorname{supp} h_{\alpha}-\operatorname{supp} F f \subset G-\operatorname{sp}(f),
\end{aligned}
$$

the Nikolsky inequality, and (36), we get $F^{-1} h_{\alpha} \in L_{\infty}, F^{-1} h_{\alpha}() f.\left(x-\right.$.) $\in L_{p}$ for any $x \in \mathbb{R}^{n}, \alpha \geq 0$, and

$$
\begin{aligned}
\left|\left(F^{-1} h_{\alpha} F f\right)(x)\right|^{p} & \leq\left(\int\left|F^{-1} h_{\alpha}(y) f(x-y)\right| d y\right)^{p} \\
& \leq C_{1}^{p} \int\left|F^{-1} h_{\alpha}(y)\right|^{p}|j(x-y)|^{p} d y,
\end{aligned}
$$

where $C_{1}=C_{1}(p, G-\operatorname{sp}(f))$. Therefore, given (36) we obtain

$$
\begin{aligned}
\left\|D^{\alpha} f\right\|_{p} & =\left\|F^{-1} h_{\alpha} F f\right\|_{p} \leq C_{1}\left\|F^{-1} h_{\alpha}\right\|_{p}\|f\|_{p} \\
& \leq C_{2}\left\|h_{\alpha}\right\|_{(k)}\|f\|_{p} \leq C\left\|h_{\alpha}\right\|_{k, 2}\|f\|_{p}
\end{aligned}
$$

for all $\alpha \geq 0$. So we have proved (35).

The proof of Theorem 1 is complete.

Remark 1. By an easier way we can prove Theorem 1 for functions defined on torus $\mathbb{T}^{n}$.

Remark 2. Theorem 1 still holds for the case of fractional derivatives. And it can be extended to the cases of other derivatives as Riesz' or Bessel's ones (see [8]).

Remark 3. Equality (1) is not true if $\operatorname{sp}(f)$ is unbounded. However, we have 
Theorem 2. Let $0<p \leq \infty, f(x) \in L_{p}\left(\mathbb{R}^{n}\right)$, and $\operatorname{sp}(f)$ with respect to $\xi_{1}, \ldots$, $\xi_{k}(1 \leq k \leq n)$ be bounded. Then $D^{\nu} f(x) \in L_{p}\left(\mathbb{R}^{n}\right)$ for all $\nu=\left(\nu_{1}, \ldots, \nu_{k}\right.$, $0, \ldots, 0) \in \mathbb{Z}_{+}^{n}$ and

$$
\lim _{|\nu| \rightarrow \infty}\left(\left\|D^{\nu} f\right\|_{p} / \sup _{\operatorname{sp}(f)}\left|\xi^{\nu}\right|\right)^{1 /|\nu|}=1
$$

\section{AN APPLICATION}

Now let us apply Theorem 1 to obtain a nonconvex case of the Paley-WienerSchwartz theorem. For this purpose we have to introduce a notion on a set generated by a number sequence: Let $0 \leq \lambda_{\alpha}<\infty, \alpha \geq 0$. Denote by $G\left\{\lambda_{\alpha}\right\}$ the set of all points $\xi \in \mathbb{R}^{n}$ such that $\left|\xi^{\alpha}\right| \leq \lambda_{\alpha}$ for all $\alpha \geq 0$. Then it is easy to see that $G\left\{\lambda_{\alpha}\right\}$ is compact and $G\left\{h^{|\alpha|} \lambda_{\alpha}\right\}=h G\left\{\lambda_{\alpha}\right\}, h \geq 0$. Note that $G\left\{\lambda_{\alpha}\right\}$ can be nonconvex. Actually, let $n=2, \lambda_{(i, j)}=2^{|i-j|}, i, j \in \mathbb{Z}_{+}$. Then $G\left\{\lambda_{(i, j)}\right\}=\left\{(x, y) \in \mathbb{R}^{2}:|x y| \leq 1,|x| \leq 2,|y| \leq 2\right\}$-the parabola cross.

If there exist $m \geq 1$ and $\beta \geq 0$ such that $\lambda_{\beta}^{m}<\lambda_{m \beta}$, then $G\left\{\lambda_{\alpha}\right\}$ does not change when we replace $\lambda_{m \beta}$ by $\lambda_{\beta}^{m}$. So, to define $G\left\{\lambda_{\alpha}\right\}$ we can always assume that the sequence $\left\{\lambda_{\alpha}\right\}$ is right, i.e. $\lambda_{\alpha} \geq \lambda_{m \alpha}^{1 / m}$ for all $\alpha \geq 0$ and $m \geq 1$. Using Theorem 1 , we can prove the following

Theorem 3. Let $0<p \leq \infty, f(x) \in L_{p}\left(\mathbb{R}^{n}\right)$ and $\left\{\lambda_{\alpha}\right\}$ be right. Then $\operatorname{sp}(f) \subset$ $G\left\{\lambda_{\alpha}\right\}$ if and only if

$$
\varlimsup_{|\alpha| \rightarrow \infty}\left(\left\|D^{\alpha} f\right\|_{p} / \lambda_{\alpha}\right)^{1 /|\alpha|} \leq 1 .
$$

Proof. Let $\operatorname{sp}(f) \subset G\left\{\lambda_{\alpha}\right\}$. Then

$$
\sup _{\mathbf{s p}(f)}\left|\xi^{\alpha}\right| \leq \lambda_{\alpha}, \quad \alpha \geq 0 .
$$

Therefore, since Theorem 1, we get (37).

Conversely, if (37) holds, then given Theorem 1 we have

$$
\varlimsup_{|\alpha| \rightarrow \infty}\left(\sup _{\operatorname{sp}(f)}\left|\xi^{\alpha}\right| / \lambda_{\alpha}\right)^{1 /|\alpha|} \leq 1 .
$$

Therefore, for any $\epsilon>0$ there exists a number $N<\infty$ such that

$$
\sup _{\operatorname{sp}(f)}\left|\xi^{\alpha}\right| \leq(1+\epsilon)^{|\alpha|} \lambda_{\alpha}, \quad|\alpha| \geq N .
$$

On the other hand, the sequence $\left\{\lambda_{\alpha}\right\}$ is right; therefore,

$$
\sup _{\operatorname{sp}(f)}\left|\xi^{\alpha}\right| \leq(1+\epsilon)^{|\alpha|} \lambda_{\alpha}
$$

for all $\alpha \geq 0$. Hence,

$$
\operatorname{sp}(f) \subset(1+\epsilon) G\left\{\lambda_{\alpha}\right\} .
$$

Letting $\epsilon \rightarrow 0$, we get $\operatorname{sp}(f) \subset G\left\{\lambda_{\alpha}\right\}$. The proof is complete.

\section{REFERENCES}

1. R. A. Adams, Sobolev spaces, Academic Press, New York, San Francisco, and London, 1975.

2. H. H. Bang, A property of infinitely differentiable functions, Proc. Amer. Math. Soc. 108 (1990), 73-76. 
3. Some imbedding theorems for the spaces of infinite order of periodic functions, Math. Notes 43 (1988), 509-517.

4. __ On imbedding theorems for Sobolev spaces of infinite order, Mat. Sb. 136 (1988), 115-127.

5. _ Imbedding theorems for Sobolev spaces of infinite order, Acta Math. Vietnam 14 (1989), 17-29.

6. Ju. B. Egorov, Lectures on partial differential equations, Moscow State Univ. Press., Moscow, 1975.

7. L. Hörmander, The analysis of linear partial differential operators I, Grundlehren Math. Wiss., 256, Springer, Berlin, Heidelberg, New York, and Tokyo, 1983.

8. P. I. Lizorkin, Estimates for trigonometric integrations and the Bernstein inequality for fractional derivatives, Izv. Akad. Nauk SSSR Ser. Mat. 29 (1965), 109-126.

9. R. J. Nessel and G. Wilmes, Nikolskii-type inequalities for trigonometric polynomials and entire functions of exponential type, J. Austral. Math. Soc. 25 (1978), 7-18.

10. S. M. Nikolsky, Approximation of functions of several variables and imbedding theorems, "Nauka", Moscow, 1977.

Institute of Mathematics, P.O. Box 631, Bo Ho, Hanoi, Vietnam

Current address: Steklov Institute of Mathematics, MIAN, ul. Vavilova 42, Moskva, GSP-1, 117966 (from November 1, 1994 to April 30, 1995)

E-mail address: hhbang@thevinh.ac.vn 